\title{
Regulation of metabolic pathway underlying anthocyanin pigmentation of barley pericarp
}

\author{
O. Shoeva ${ }^{1 *}$, T. Kukoeva ${ }^{1}$, E. Gordeeva ${ }^{1}$, A. Glagoleva ${ }^{1}$, E. Khlestkina ${ }^{1,2,3}$ \\ ${ }^{1}$ Institute of Cytology and Genetics SB RAS, Novosibirsk, Russia \\ ${ }^{2}$ Novosibirsk State University, Novosibirsk, Russia \\ ${ }^{3}$ N.I. Vavilov All-Russian Research Institute of Plant Genetic Resources (VIR), Saint-Petersburg, Russia \\ *e-mail: olesya_ter@bionet.nsc.ru
}

Key words: Hordeum vulgare, pericarp, structural genes, regulatory genes, transcription

Motivation and Aim: Anthocyanins are secondary metabolites determining pigmentation in plants. These molecules and their uncolored precursors have important physiological roles including regulation of plant growth and development and adaptation to stress [1]. Two groups of genes underlay anthocyanin biosynthesis (AB): structural genes, encoding enzymes of the pathway, and regulatory genes, encoding MYB, bHLH or WD40 transcriptional factors (TFs). The TFs form complex that activates transcription of the structural genes. The interaction between regulatory genes at transcriptional level has been recently observed in wheat [2]. The aim of the current study was to reveal possible interactions between the genes underlying the pigmentation in barley.

Methods and Algorithms: The Ant1 (encodes the MYB TF, chromosome 7H) and Ant2 (bHLH, 2H) genes, predetermining purple pigmentation of barley pericarp, were sequenced in Bowman near-isogenic lines (NILs) differing by grain color. Primers distinguishing the dominant and recessive alleles of the genes were designed by OLIGO [3] and used for marker-assisted selection of lines with different combinations of the Ant genes (Ant1Ant1ant2ant2 and ant1 ant1Ant2Ant2). Expression of the structural (Chs, Chi, F3h, F3'h, Dfr, Ans) and regulatory (Ant1, Ant2) genes in pericarp of the NILs was determined by qPCR or qRT-PCR.

Results: We showed that the dominant and recessive alleles of the Ant genes have differences in regulatory regions that prevent expression of the recessive alleles in non-colored grain pericarp. The NILs with different combination of the dominant and recessive Ant 1 and Ant2 genes were developed based on the Bowman NILs. Transcription activity assay of the structural and regulatory genes in the NILs revealed that (1) the dominant alleles of Ant1 and Ant 2 genes are required for activation of the structural genes expression in purple pericarp and (2) Ant 1 affects expression of Ant2: transcription of Ant 2 was up-regulated in the presence of dominant Ant 1 only, whereas Ant 2 was not expressed when ant 2 was recessive.

Conclusion: The data on revealed interaction between $\mathrm{AB}$ regulatory genes is base for further genetic regulatory network reconstruction.

Acknowledgements: Supported by the RFBR (No. 16-34-60052).

\section{References}

1. Lev-Yadun S., Gould K.S. (2008) Role of anthocyanins in plant defence. In: Winefield C., Davies K., Gould K. (eds), Anthocyanins. Springer New York. pp. 22-28.

2. Gordeeva E.I. et al. (2015) Marker-assisted development of bread wheat near-isogenic lines carrying various combinations of $P p$ (purple pericarp) alleles. Euphitica. 203:469-476.

3. Offerman J.D., Rychlik W. (2003) Oligo primer analysis software. In: Krawetz S.A., Womble D.D. (eds), Introduction to Bioinformatics: a Theoretical and Practical Approach. Humana Press; New Jersey, USA. pp. 345-361. 